\title{
The visible environment of galaxies with counterrotation
}

\author{
D. Bettoni ${ }^{1}$, G. Galletta ${ }^{2}$, and F. Prada ${ }^{3}$ \\ 1 Osservatorio Astronomico di Padova, Vicolo dell'Osservatorio 5, 35122 Padova, Italy \\ 2 Dipartimento di Astronomia, Università di Padova, Vicolo dell'Osservatorio 2, 35122 Padova, Italy \\ 3 Centro Astronómico Hispano-Alemán, Apartado 511, 04080 Almería, Spain
}

Received 6 February 2001 / Accepted 23 April 2001

\begin{abstract}
In this paper we present a statistical study of the environments of 49 galaxies in which there is gasor stellar-counterrotation. The number of possible companions in the field (to apparent magnitude 22), their size and concentration were considered. All the statistical parameters were analysed by means of Kolgomorov-Smirnov tests, using a control sample of 43 galaxies without counterrotation. From our data, no significant differences between the counter-rotating and control samples appear. This is different to Seyfert or radio-loud galaxies which lie in environments with a higher density of companions. On the contrary, if a weak tendency exists, for galaxies with gas counterrotation only, it is discovered in regions of space where the large scale density of galaxies is smaller. Our results tend to disprove the hypothesis that counterrotation and polar rings derive from a recent interaction with a small satellite or a galaxy of similar size. To a first approximation, they seem to follow the idea that all galaxies are born through a merger process of smaller objects occurring very early in their life, or that they derive from a continuous, non-traumatic infall of gas that formed stars later. Whatever the special machinery is which produces counterrotation or polar rings instead of a co-planar, co-rotating distribution of gas and stars, it seems not to be connected to the present galaxy density of their environments.
\end{abstract}

Key words. galaxies: evolution - galaxies: formation - galaxies: interactions - galaxies: peculiar

\section{Introduction}

In a previous paper (Brocca et al. 1997, Paper I) we studied the environment of galaxies circled by a polar ring of gas and stars, a kind of stellar system whose origin is still under discussion: in recent theoretical works the polar rings are obtained by means of a merging of galaxies (Bekki 1998), whereas in other studies, it is ascribed either to the accretion of matter during a close encounter with a nearby donor galaxy (Reshetnikov \& Sotnikova 1997), or to the capture of gas or stars from the environment (Whitmore et al. 1990; Tremaine \& Yu 2000). It is evident that in the three suggested scenarios, the present environment of these galaxies should appear different in the richness of small satellites or bright companions. For instance, in the hypothesis of a close encounter, the donor galaxy should not be too far from the polar ring galaxy, whereas in the case of diffuse gas, no visible differences are expected. The epoch of the "second event" may also play a role in the possibility of detecting the donor galaxy. From the data of Paper I, we concluded that the environment of polar ring galaxies appears to be similar to that of normal galaxies in relation to the richness, density or

Send offprint requests to: G. Galletta, e-mail: galletta@pd.astro.it concentration of satellites. We deduced that this result favours the infall of matter or a very early merger theory.

A different result has been found by analysing the environment of other galaxy categories, whose peculiar origin has been connected in the literature to galactic interactions or mergers (Gunn 1979), similar to polar rings. In particular, the environment of Seyfert galaxies appears to be richer in physical companions than that of normal galaxies (Dahari 1984; Rafanelli et al. 1995). Similarly, the local galaxy density of 47 radio-loud elliptical and lenticular galaxies appears higher than that of radio-quiet galaxies by a factor 2-3 (Heckman et al. 1985). Even radioloud QSO with $0.9<z<1.5$ appear surrounded by a statistically significant excess of galaxies (Hintzen et al. 1991). Among these peculiar environments, the apparent normality of polar ring galaxies looks anomalous and distinguishes them from the other categories of astronomical objects whose peculiarity or activity is ascribed to interaction or merger. Therefore, we wish to see if other types of peculiar galaxies have an environment similar to that of the active galaxies or if they are "normal" in this respect, as the polar ring galaxies appear to be.

In this paper, the environment of another category of peculiar galaxies, in which the rotation of the gas or of the stars is opposite to that of most of the stars in the galaxy, 
is analysed. This phenomenon, known as "counterrotation", presents a variety of aspects: it may be present in the gas only (Bettoni 1984; Caldwell et al. 1986; Galletta 1987; Ciri et al. 1995), in a portion of the stars (Bender 1988; Franx 1988; Jedrzejewski \& Schecther 1988; Bettoni 1989; Rubin et al. 1992; Merrifield and Kuijken 1994; Prada et al. 1996, 1999) or in both (Bertola et al. 1996; Galletta 1987). The existing observations suggest that counterrotation is a phenomenon which is present all along the Hubble sequence. As in the case of polar ring galaxies, the origin of the counterrotation has been attributed to different mechanisms. One of these is the collision between the accretor galaxy and a small satellite (Kennicut 1996; Thakar \& Ryden 1996). The difference to the formation of a polar ring may reside in the satellite orbit, coplanar with the disk of the accretor (and retrograde) to generate counterrotation (Thakar \& Ryden 1996). Another mechanism may be the merger of two spirals of unequal mass (Balcells \& Gonzalez 1998; Bekki 1998), which is able to transfer matter as large as $10^{8} M_{\odot}$ or more in counterrotation (Bettoni et al. 1991; Rubin et al. 1992; Ciri et al. 1995; Bettoni et al. 1999). A third alternative involves an extended period of star- or gas-infall during which the spin of the accreted matter changes rapidly (Voglis et al. 1991; Quinn \& Binney 1992; Merrifield \& Kuijken 1994; Ostriker \& Binney 1989; Rix et al. 1992).

If counterrotation arises from accretion of a satellite galaxy, there should be some peculiarity in the environment of these galaxies that may be detectable in the present epoch, as happens in Seyfert or radio-loud galaxies. Possibilities include: 1) a local over-density of galaxies or 2) a larger number of satellites if the accretion event occurred recently. A trace of the satellite accretion may also remain if the phenomenon is not recent; for instance, a deficit of satellites due to depletion of the environment may differentiate them from a normal population. Finally, if the counterrotation is generated by pure gas, pure star infall or by a merger in the early epoch of galaxy formation, no traces of differences should exist in the present surrounding field.

\section{Selection of the samples}

The first step in this work was to select a sample of galaxies with counterrotation and, as wide as possible, a comparison sample of "normal" galaxies. The latter should be representative of galaxies without counterrotation but with distributions of luminosity and morphological type similar to that of the galaxies with counterrotation. This selection required a short analysis of the samples.

\subsection{Galaxies with counterrotation}

Our initial selection from the literature included all known examples of gas or stellar counterrotation. For this selection the compilations of Galletta (1996) and Corsini \& Bertola (1998) were used. We removed from the lists a few doubtful examples and those with still unpublished references. These included NGC 2217, with a polar ring seen almost face-on (Bettoni et al. 1990) and NGC 4684, with gas streams along a pole-on bar (Bettoni et al. 1993) that mimic gas counterrotation. At the end of this selection, our working list contained 49 galaxies, whose names are indicated in the first column of Table 2. They have been divided according to the kind of counterrotation present. Four systems show both kinds of counterrotation and have been considered separately in the statistical analysis. They include IC $4889=$ IC 4891, NGC 3593, NGC 4550 and NGC 7079. In the following, the label "gas cr" or "stars cr" refer only to the pure cases of gas and star counterrotation, while "all cr" or "cr galaxies" indicates both samples plus these four galaxies.

The mean astrophysical parameters of these galaxies have been obtained from the Lyon-Meudon Extragalactic Database (Paturel et al. 1997). They are apparent and absolute magnitudes, radial velocity and morphological type.

\subsection{Selection of the comparison galaxies}

In order to prepare a comparison sample, we made an initial list of galaxies for which both gas and star rotation curves were published. The principal source for this list was the Catalogue of Spatially Resolved Kinematics of Galaxies (Prugniel et al. 1998) that is available on the web. The published rotation curves for each galaxy in the list was analysed, in order to determine the data-supported co-rotation for the stars and gas. The comparison sample also includes galaxies studied by Kuijken et al. (1996), where the authors claim that no counter-rotating cores have been detected.

This list contains a high percentage of spirals, whereas galaxies with counterrotation mostly have morphological types earlier than Sa. This may alter the comparison between samples, with spiral galaxies generally being present in a lower density environment. To minimize this difference, we extracted from the initial list of comparison galaxies a sub-sample with a distribution of morphological types similar to that of galaxies with counterrotation. This sub-sample was checked to exclude the presence of other biases in their general properties, applying a Kolgomorov-Smirnov test to their distributions of absolute magnitudes $M_{B}$, red-shift and morphological type. To this end, we determined the cumulative frequency distribution $S(X)$ for each sample of observations by using the same interval for both distributions. $S(X)$ is the fraction of data observed equal to or less than $X$. Then, for each interval we subtracted one step function from the other, evaluating $D_{\alpha}=\max \left|S_{1}(X)-S_{2}(X)\right|$ which is the maximum absolute difference found for the two distributions. The probability of the two samples having the calculated $D_{\alpha}$ and coming from different galaxy populations is estimated by means of the theoretical significance level SL, which is a function of the sizes of the samples and is tabulated in statistical books. Comparing various sub-samples of comparison galaxies we found one whose distribution of 
intrinsic properties is not significantly different, at a confidence level of $S L=95 \%$, from that of all the cr galaxies (see Table 1). All the $S L$ values calculated on the basis of the corresponding $D_{\alpha}$ are lower than this value.

An independent test of the quality of this comparison sample concerns the large scale environment in which these galaxies are located with respect to that of the $\mathrm{cr}$ galaxies. We then checked each galaxy for which kind of environment it belongs to: field, small groups and large clusters. This classification was made for galaxies with redshift $\leq 3000 \mathrm{~km} \mathrm{~s}^{-1}$ by Tully (1987) who indicates for each one the richness and the velocity dispersion of the group to which it belongs. The group richness is also available for galaxies brighter than magnitude 14 and with red-shift lower than $5500 \mathrm{~km} \mathrm{~s}^{-1}$ (Garcia et al. 1993).

These data show that the galaxies with counterrotation are present in all kinds of environments, discovered both in clusters as rich as Virgo as well in regions of the sky apparently empty of companions. The distributions of richness and group velocity dispersion show a large spread of values, without any tendency for clustering of data at particular values. As performed for the absolute magnitudes and morphological types, a Kolgomorov-Smirnov test was applied to the samples (see Table 1) and indicates that the differences between galaxies with counterrotations and the comparison sample are not significant.

These tests defined the final list for the comparison galaxies that was used in the following sections as a reference sample of normal galaxies. It is shown in the first column of Table 3 and contains 43 galaxies.

\section{Data production and analysis}

To study the properties of the visible environment of these galaxies, we searched all the objects present in the sky around every galaxy, starting from the optical image databases available in the literature. Our research was focused along different lines:

1) a first search of faint objects in the close neighbourhood of each galaxy;

2) a second search of bright objects that may have encountered the galaxy within the last billion years;

3) a comparison of the density of galaxies within $40 \mathrm{Mpc}$, taken from the literature (Tully 1988), taking into account the presence of the sample galaxies in groups of different hierarchy. This test is an extension of that discussed in Sect. 2.2.

In the following, the galaxy to be studied, located at the centre of each field, will be referred to as the "central galaxy", whereas all the objects present in the selected field will be called "nearby objects", even if they are in the foreground or in the background with respect to the central galaxy. Only when the red-shift difference between the nearby object and the central galaxy is lower than a fixed value, described subsequently, will the object be defined as a "companion".

\subsection{Search for faint objects}

The first search was performed by adopting a searching radius of $100 \mathrm{kpc}$ and looking at all the objects present within that radius. The search was performed by extracting the data from the APM Sky Catalogue, available for Internet access from the Observatory of Edinburgh (Irwing et al. 1994). It contains data extracted by scanning and photometrically calibrating the $B$ and $R$ plates of the Palomar Sky Survey and the ESO/SRC J survey. It lists all the objects present in the plates over the brightness level of $24 \mathrm{mag} \operatorname{arcsec}^{-2}$ for the blue plates and $23 \mathrm{mag} \mathrm{arcsec}^{-2}$ for the red plates. They correspond to a limiting apparent magnitude $B=21.5$ and $R=20.0$. For each object present in a field corresponding to $100 \mathrm{kpc}$ at the distance of the central galaxy, we extracted the following parameters: $\alpha$ and $\delta$ co-ordinates, $B$ and $R$ apparent magnitudes, semi-major axis, ellipticity and PA of the ellipse fitting the image. In APM, galaxies are distinguished from stars by means of a comparison of their Point Spread Function with that of an "average" stellar image.

For most of the objects in the field the true distance is unknown, so we adopted the following method to discard background and foreground objects: all the parameters extracted from APM were converted in distance from the central galaxy $(\mathrm{kpc})$, absolute magnitude and linear size (kpc) as if all the objects were at the same distance as the central galaxy. We expected that many background galaxies would appear with a linear size or magnitude too small to be real companions. Similarly, eventual foreground galaxies would appear too big. The limits taken to keep a galaxy were from 2 to $50 \mathrm{kpc}$ for the size and from $M_{B}=-14$ to $M_{B}=-23$. These figures have been chosen as typical mean size and luminosity of the galaxies, taken from the Local Group members (Zombeck 1990; Sandage \& Tamman 1981) and from the Revised ShapleyAmes Catalog (Sandage \& Tamman 1981). A limit of this method is given by the fact that galaxies intrinsically fainter than $M_{B}=-14$ do exist, e.g. Leo I, whose absolute magnitude is $M_{B}=-9.6$ (Sandage \& Tamman 1981). It is clear that a more relaxed limit, for instance $M_{B} \leq-9$, may include all the possible dwarf galaxies in the surroundings of the central galaxy but will surely fill the sample of nearby objects with a large number of background galaxies. After a set of tests with nearby central galaxies, we chose to limit the sample at $M_{R}=-14$, bearing in mind that for fainter limits the possibility of contamination by background objects is higher than the chances of excluding possible dwarfs. Based on the distances of the central object, we computed that an object of $M_{B}=-14$ with apparent $B$ magnitudes should always appear brighter than the APM detection limits both for counterrotation and normal galaxy fields. For this reason, we are confident that most of the faint objects around the sample galaxies are present in our search.

A problem to be faced in using the APM data is that the large galaxies present in the field, especially if belonging to late morphological types, appear fragmented in a set 
Table 1. Summary of the Kolgomorov-Smirnov tests made to choose a comparison sample of normal galaxies. As explained in the text, $D_{\alpha}$ is the maximum fractional difference observed between the two distributions, while $S L$ is the significance level at which the two distributions compared are different.

\begin{tabular}{|c|c|c|c|c|c|c|}
\hline \multirow[b]{2}{*}{ Parameter } & \multicolumn{2}{|c|}{ gas cr vs. no cr } & \multicolumn{2}{|c|}{ star cr vs. no cr } & \multicolumn{2}{|c|}{ all $\mathrm{cr}$ vs. no $\mathrm{cr}$} \\
\hline & $D_{\alpha}$ & $S L$ & $D_{\alpha}$ & $S L$ & $D_{\alpha}$ & $S L$ \\
\hline Morphological Type & 0.344 & $92.3 \%$ & 0.220 & $57.1 \%$ & 0.240 & $84.2 \%$ \\
\hline$M_{B}$ & 0.244 & $60.7 \%$ & 0.152 & $13.5 \%$ & 0.170 & $44.2 \%$ \\
\hline Red-shift & 0.314 & $86.6 \%$ & 0.193 & $40.0 \%$ & 0.222 & $77.1 \%$ \\
\hline N. members (Garcia et al. 1993) & 0.175 & $<10 \%$ & 0.157 & $<10 \%$ & 0.133 & $<10 \%$ \\
\hline N. members (Tully 1987) & 0.218 & $22.9 \%$ & 0.034 & $<10 \%$ & 0.118 & $<10 \%$ \\
\hline Group velocity dispersion (Tully 1987) & 0.096 & $<10 \%$ & 0.112 & $<10 \%$ & 0.058 & $<10 \%$ \\
\hline
\end{tabular}

of small extended objects, reducing their contribution to the local population of galaxies extracted from APM. For this reason, we had to look at all the fields by plotting the position and size of the objects present around the central galaxies and we compared this map with the image of the same field extracted from Palomar or ESO/SRJ atlases. When a galaxy is not included in the APM catalogue, we extracted its position and size from other catalogues and inserted it in our files. At the end of this correction, our set of data contained 40 galaxies with counterrotation and 38 comparison galaxies, some systems not being present in the APM catalogue. There are 16 galaxies with pure gas counterrotation, whereas 20 systems exhibit pure stellar counterrotation. Four galaxies have both types of counterrotation.

\subsection{Search for bright companions}

A second search concerned the detection of bright galaxies that may have been gas or star donors during a close encounter. In this case the search area has been defined in a different way: assuming that a companion galaxy exists which may have encountered the central galaxy in the past, its linear distance will be $R=\Delta V \cdot \Delta t$, $\Delta V$ being the relative velocity in space and $\Delta t$ the time elapsed since the encounter. If we assume a typical maximum value $\Delta V=600 \mathrm{kms}^{-1}$ and a maximum elapsed time of $1 \mathrm{Gyr}$, the maximum projected angular distance between the two galaxies seen at a distance $d$ will be $R_{\max }[\operatorname{arcmin}]=2110.8 / d[\mathrm{Mpc}]$, which was our search radius. This corresponds to a linear distance of $0.61 \mathrm{Mpc}$. We then searched galaxies within a radius of $0.61 \mathrm{Mpc}$ from the central galaxy and having red-shift difference $c z$ lower than $600 \mathrm{~km} \mathrm{~s}^{-1}$ with respect to it.

This time we used the NED database, extracting position, red-shift, apparent magnitude and size of every listed galaxy lying on the sky inside $R_{\max }$ and having $\Delta V \leq$ $600 \mathrm{~km} \mathrm{~s}^{-1}$. These data were converted into distances from the central galaxy, differences in radial velocity, absolute magnitudes and linear sizes. In this search, only galaxies with known redshift were included in the sample. The final set of data includes 47 galaxies with counterrotation (18 gas cr, 25 star cr, 4 mixed) and 42 galaxies of the comparison sample. The excluded objects have too wide a field (such as NGC 253, with 14.6 of search field) or have no published red-shift (e.g. NGC 2612).

\subsection{Large scale environment}

In addition to our data, we also used density values present in the literature and computed on a much wider scale. We extracted from the Nearby Galaxies Catalog (Tully 1988) the values of $\rho_{x y z}$, the density of galaxies brighter than -16 mag determined within $40 \mathrm{Mpc}$ using a grid of $0.5 \mathrm{Mpc}$.

To have a direct comparison with our data, a "galaxy density" from APM and NED data was calculated. In the first case, the total number of observed faint objects within $100 \mathrm{kpc}$ has produced $\rho_{\mathrm{APM}}$, the projected density of faint galaxies in units of $\mathrm{Mpc}^{-2}$. The area of $1 \mathrm{Mpc}^{2}$ is merely an arbitrary choice to plot the data and produce large numbers. In the second case, the NED data were used to produce a $\rho_{\mathrm{NED}}$, in galaxies $/ \mathrm{Mpc}^{3}$, corresponding to the mean density of bright objects with known redshift present in a sphere of volume $0.95 \mathrm{Mpc}^{3}$, whose radius is the distance covered at a velocity of $600 \mathrm{~km} \mathrm{~s}^{-1}$ in $1 \mathrm{Gyr}$.

The values so determined from our data are listed in the last columns of Tables 2 and 3 and are plotted in the two panels of Fig. 1 versus $\rho_{x y z}$.

\section{Statistical parameters}

The statistical analysis of all fields for the cr galaxies and comparison sample was done defining a set of density parameters for each field:

$\rho_{i j}=\sum_{k} r_{k}^{-i} D_{k}^{j}$

where $r_{k}$ is the projected distance between the central galaxy and the $k$ th galaxy, $D_{k}$ is the projected diameter of the $k$ th galaxy and $(i, j)$ assumes the values $0,1,(2,2)$ and $(3,2.4)$. We normalised the $D_{k}$ and $r_{k}$ values in units of $100 \mathrm{kpc}$.

The first three parameters describe the environment of the galaxies with different criteria: the population $\left(\rho_{00}\right)$, the total size of sky covered by surrounding galaxies $\left(\rho_{01}\right)$, the concentration of surrounding galaxies $\rho_{10}$. The remaining three are linked with these: $\rho_{11}$ is proportional 
Table 2. Statistical parameters $\rho_{i j}$ and galaxy densities for galaxies with counterrotation.

\begin{tabular}{|c|c|c|c|c|c|c|c|c|c|c|c|c|c|c|c|}
\hline \multirow[t]{2}{*}{ Name } & \multicolumn{6}{|c|}{ APM data } & \multicolumn{6}{|c|}{ NED data } & \multicolumn{3}{|c|}{ galaxy densities } \\
\hline & $\rho_{00}$ & $\rho_{01}$ & $\rho_{10}$ & $\rho_{11}$ & $\rho_{22}$ & $\rho_{3,2.4}$ & $\rho_{00}$ & $\rho_{01}$ & $\rho_{10}$ & $\rho_{11}$ & $\rho_{22}$ & $\rho_{3,2.4}$ & $\rho_{x y z}$ & $\rho_{\mathrm{NED}}$ & $\rho_{\mathrm{APM}}$ \\
\hline \multicolumn{16}{|c|}{ gas cr } \\
\hline ESO 263-G48 & & & & & & & 1 & 0.168 & 0.084 & 0.014 & 0.000 & 0.000 & & 1 & \\
\hline IC 2006 & & & & & & & 14 & 1.347 & 4.680 & 0.412 & 0.028 & 0.014 & & 15 & \\
\hline NGC 128 & 3 & 0.264 & 15.538 & 1.220 & 0.568 & 1.328 & 10 & 1.842 & 17.609 & 2.354 & 1.677 & 5.188 & & 11 & 100 \\
\hline NGC 253 & 0 & 0.000 & 0.000 & 0.000 & 0.000 & 0.000 & & & & & & & 0.22 & & 0 \\
\hline NGC 497 & 5 & 0.363 & 6.457 & 0.483 & 0.061 & 0.034 & 3 & 0.663 & 0.580 & 0.120 & 0.005 & 0.001 & & 3 & 167 \\
\hline NGC 1052 & 7 & 0.506 & 17.999 & 0.930 & 0.209 & 0.273 & 9 & 1.408 & 4.744 & 0.696 & 0.123 & 0.076 & 0.49 & 9 & 234 \\
\hline NGC 1216 & & & & & & & 2 & 0.584 & 2.839 & 0.857 & 0.470 & 0.584 & & 2 & \\
\hline NGC 2768 & 2 & 0.147 & 2.472 & 0.174 & 0.015 & 0.006 & 2 & 0.239 & 0.731 & 0.076 & 0.003 & 0.000 & 0.31 & 2 & 67 \\
\hline NGC 3497 & 9 & 0.389 & 15.892 & 0.667 & 0.077 & 0.059 & 1 & 0.142 & 1.022 & 0.146 & 0.021 & 0.010 & & 1 & 300 \\
\hline NGC 3626 & 2 & 0.080 & 4.148 & 0.193 & 0.028 & 0.025 & 10 & 0.984 & 5.632 & 0.518 & 0.051 & 0.022 & 0.32 & 11 & 67 \\
\hline NGC 3941 & 1 & 0.041 & 1.650 & 0.067 & 0.005 & 0.002 & 3 & 0.374 & 0.804 & 0.103 & 0.004 & 0.001 & 0.29 & 3 & 34 \\
\hline NGC 4379 & 3 & 0.152 & 4.801 & 0.263 & 0.036 & 0.026 & 34 & 2.964 & 10.198 & 0.927 & 0.055 & 0.014 & 2.89 & 36 & 100 \\
\hline NGC 4546 & 2 & 0.071 & 6.439 & 0.209 & 0.023 & 0.021 & 2 & 0.151 & 0.541 & 0.042 & 0.001 & 0.000 & 0.27 & 2 & 67 \\
\hline NGC 4826 & 1 & 0.023 & 1.299 & 0.029 & 0.001 & 0.000 & & & & & & & 0.20 & & 34 \\
\hline NGC 5252 & 5 & 0.214 & 7.941 & 0.358 & 0.031 & 0.018 & 1 & 0.242 & 0.272 & 0.066 & 0.004 & 0.001 & & 1 & 167 \\
\hline NGC 5354 & 6 & 0.525 & 23.129 & 2.038 & 1.301 & 3.848 & 15 & 2.801 & 12.884 & 2.455 & 1.076 & 1.519 & & 16 & 200 \\
\hline NGC 5898 & & & & & & & 5 & 0.788 & 6.385 & 0.856 & 0.252 & 0.292 & 0.23 & 5 & \\
\hline NGC 7007 & 7 & 0.241 & 8.932 & 0.298 & 0.013 & 0.004 & 0 & 0.000 & 0.000 & 0.000 & 0.000 & 0.000 & 0.14 & 0 & 234 \\
\hline NGC 7097 & 8 & 0.245 & 11.739 & 0.385 & 0.026 & 0.014 & 2 & 0.197 & 0.697 & 0.060 & 0.002 & 0.000 & 0.26 & 2 & 267 \\
\hline NGC 7332 & 3 & 0.174 & 11.996 & 0.629 & 0.222 & 0.334 & 1 & 0.136 & 3.310 & 0.451 & 0.204 & 0.304 & 0.12 & 1 & 100 \\
\hline
\end{tabular}

\begin{tabular}{|c|c|c|c|c|c|c|c|c|c|c|c|c|c|c|c|}
\hline \multicolumn{16}{|c|}{ stars cr } \\
\hline IC 1459 & 9 & 0.586 & 15.543 & 0.936 & 0.205 & 0.186 & 14 & 2.033 & 2.529 & 0.400 & 0.026 & 0.006 & 0.28 & 15 & 300 \\
\hline NGC 936 & 3 & 0.199 & 5.944 & 0.332 & 0.042 & 0.025 & 5 & 0.580 & 3.792 & 0.439 & 0.062 & 0.038 & 0.24 & 5 & 100 \\
\hline NGC 1439 & & & & & & & 12 & 1.469 & 3.114 & 0.387 & 0.017 & 0.003 & 0.45 & 13 & \\
\hline NGC 1543 & 0 & 0.000 & 0.000 & 0.000 & 0.000 & 0 & 11 & 1.288 & 1.983 & 0.211 & 0.004 & 0.000 & 0.95 & 12 & 0 \\
\hline NGC 1574 & 1 & 0.021 & 1.828 & 0.039 & 0.002 & 0.001 & 20 & 2.376 & 4.221 & 0.520 & 0.022 & 0.003 & 0.98 & 21 & 34 \\
\hline NGC 1700 & & & & & & & 0 & 0.000 & 0.000 & 0.000 & 0.000 & 0.000 & & 0 & \\
\hline NGC 2841 & 1 & 0.021 & 2.288 & 0.049 & 0.002 & 0.001 & 3 & 0.104 & 1.912 & 0.062 & 0.002 & 0.000 & 0.13 & 3 & 34 \\
\hline NGC 2983 & & & & & & & 1 & 0.250 & 0.215 & 0.054 & 0.003 & 0.000 & 0.16 & 1 & \\
\hline NGC 3608 & 4 & 0.199 & 11.118 & 0.524 & 0.083 & 0.087 & 12 & 0.988 & 11.485 & 1.283 & 0.534 & 0.869 & 0.56 & 13 & 134 \\
\hline NGC 4073 & 9 & 0.592 & 12.717 & 0.805 & 0.104 & 0.06 & 10 & 1.946 & 4.652 & 0.758 & 0.080 & 0.031 & & 11 & 300 \\
\hline NGC 4138 & 3 & 0.088 & 3.942 & 0.117 & 0.005 & 0.002 & 14 & 1.494 & 5.162 & 0.562 & 0.043 & 0.014 & 0.84 & 15 & 100 \\
\hline NGC 4472 & 6 & 0.180 & 11.165 & 0.345 & 0.023 & 0.013 & 71 & 4.502 & 46.441 & 2.437 & 0.235 & 0.283 & 3.31 & 75 & 200 \\
\hline NGC 4477 & 4 & 0.213 & 7.383 & 0.386 & 0.053 & 0.041 & 39 & 3.283 & 13.461 & 1.222 & 0.106 & 0.053 & 4.06 & 41 & 134 \\
\hline NGC 4596 & 2 & 0.064 & 2.632 & 0.086 & 0.004 & 0.002 & 12 & 1.574 & 4.078 & 0.505 & 0.041 & 0.013 & 2.87 & 13 & 67 \\
\hline NGC 4643 & 0 & 0.000 & 0.000 & 0.000 & 0.000 & 0 & 18 & 2.700 & 3.436 & 0.522 & 0.030 & 0.006 & 0.25 & 19 & 0 \\
\hline NGC 4816 & 6 & 0.328 & 9.335 & 0.528 & 0.069 & 0.05 & 26 & 4.333 & 9.467 & 1.505 & 0.359 & 0.464 & & 27 & 200 \\
\hline NGC 5005 & 0 & 0.000 & 0.000 & 0.000 & 0.000 & 0 & 8 & 0.796 & 1.919 & 0.210 & 0.015 & 0.003 & 0.28 & 8 & 0 \\
\hline NGC 5322 & 7 & 0.219 & 11.397 & 0.337 & 0.020 & 0.011 & 9 & 1.344 & 1.211 & 0.153 & 0.003 & 0.000 & 0.43 & 9 & 234 \\
\hline NGC 5728 & 6 & 0.175 & 8.930 & 0.287 & 0.022 & 0.016 & 0 & 0.000 & 0.000 & 0.000 & 0.000 & 0.000 & 0.18 & 0 & 200 \\
\hline NGC 6684 & & & & & & & 7 & 1.137 & 0.736 & 0.092 & 0.002 & 0.000 & 0.21 & 7 & \\
\hline NGC 6701 & 15 & 0.549 & 27.578 & 0.977 & 0.082 & 0.058 & 0 & 0.000 & 0.000 & 0.000 & 0.000 & 0.000 & & 0 & 500 \\
\hline NGC 7217 & 0 & 0.000 & 0.000 & 0.000 & 0.000 & 0 & 0 & 0.000 & 0.000 & 0.000 & 0.000 & 0.000 & 0.15 & 0 & 0 \\
\hline NGC 7331 & & & & & & & 3 & 0.203 & 1.089 & 0.066 & 0.002 & 0.000 & 0.33 & 3 & \\
\hline NGC 7796 & 6 & 0.415 & 10.085 & 0.718 & 0.132 & 0.113 & 0 & 0.000 & 0.000 & 0.000 & 0.000 & 0.000 & & 0 & 200 \\
\hline UGC 9922A & 8 & 0.287 & 12.187 & 0.445 & 0.029 & 0.014 & 1 & 0.043 & 4.005 & 0.174 & 0.030 & 0.034 & & 1 & 267 \\
\hline \multicolumn{16}{|c|}{ gas plus stars cr } \\
\hline IC 4889 & 10 & 0.410 & 16.651 & 0.680 & 0.060 & 0.033 & 1 & 0.108 & 0.315 & 0.034 & 0.001 & 0.000 & 0.13 & 1 & 334 \\
\hline NGC 3593 & 0 & 0.000 & 0.000 & 0.000 & 0.000 & 0 & 8 & 1.159 & 3.301 & 0.592 & 0.093 & 0.036 & 0.19 & 8 & 0 \\
\hline NGC 4550 & 2 & 0.080 & 7.225 & 0.348 & 0.095 & 0.161 & 7 & 0.315 & 7.640 & 0.396 & 0.053 & 0.050 & 2.97 & 7 & 67 \\
\hline NGC 7079 & 0 & 0.000 & 0.000 & 0.000 & 0.000 & 0 & 1 & 0.166 & 0.241 & 0.040 & 0.002 & 0.000 & 0.19 & 1 & 0 \\
\hline
\end{tabular}


Table 3. Statistical parameters $\rho_{i j}$ and galaxy densities for the galaxies of the comparison sample.

\begin{tabular}{|c|c|c|c|c|c|c|c|c|c|c|c|c|c|c|c|}
\hline \multirow[t]{2}{*}{ Name } & \multicolumn{6}{|c|}{ APM data } & \multicolumn{6}{|c|}{ NED data } & \multicolumn{3}{|c|}{ galaxy densities } \\
\hline & $\rho_{00}$ & $\rho_{01}$ & $\rho_{10}$ & $\rho_{11}$ & $\rho_{22}$ & $\rho_{3,2.4}$ & $\rho_{00}$ & $\rho_{01}$ & $\rho_{10}$ & $\rho_{11}$ & $\rho_{22}$ & $\rho_{3,2.4}$ & $\rho_{x y z}$ & $\rho_{\mathrm{NED}}$ & $\rho_{\mathrm{APM}}$ \\
\hline IC 5063 & 9 & 0.267 & 20.083 & 0.625 & 0.055 & 0.041 & 2 & 0.290 & 0.227 & 0.037 & 0.001 & 0.000 & & 2 & 300 \\
\hline NGC 488 & 7 & 0.380 & 11.232 & 0.599 & 0.056 & 0.032 & 3 & 0.291 & 2.543 & 0.200 & 0.015 & 0.006 & 0.28 & 3 & 234 \\
\hline NGC 628 & 0 & 0.000 & 0.000 & 0.000 & 0.000 & 0 & 7 & 0.553 & 2.422 & 0.204 & 0.012 & 0.003 & 0.18 & 7 & 0 \\
\hline NGC 1023 & 2 & 0.069 & 2.261 & 0.078 & 0.003 & 0.001 & 9 & 0.625 & 17.799 & 0.833 & 0.346 & 1.328 & 0.57 & 9 & 67 \\
\hline NGC 1275 & & & & & & & 8 & 1.754 & 4.078 & 0.911 & 0.156 & 0.078 & & 8 & \\
\hline NGC 1291 & 2 & 0.053 & 5.320 & 0.147 & 0.013 & 0.01 & 3 & 0.270 & 0.910 & 0.091 & 0.004 & 0.001 & 0.14 & 3 & 67 \\
\hline NGC 1300 & & & & & & & 4 & 0.395 & 1.669 & 0.187 & 0.015 & 0.006 & 0.71 & 4 & \\
\hline NGC 1566 & 2 & 0.063 & 3.283 & 0.104 & 0.006 & 0.002 & 12 & 2.160 & 2.320 & 0.407 & 0.017 & 0.002 & 0.92 & 13 & 67 \\
\hline NGC 2613 & & & & & & & 1 & 0.124 & 0.169 & 0.021 & 0.000 & 0.000 & 0.15 & 1 & \\
\hline NGC 2787 & 4 & 0.121 & 5.705 & 0.184 & 0.012 & 0.006 & 3 & 0.173 & 0.750 & 0.038 & 0.001 & 0.000 & 0.06 & 3 & 134 \\
\hline NGC 2903 & 2 & 0.052 & 2.081 & 0.054 & 0.002 & 0 & 0 & 0.000 & 0.000 & 0.000 & 0.000 & 0.000 & 0.12 & 0 & 67 \\
\hline NGC 2974 & 5 & 0.112 & 9.964 & 0.220 & 0.012 & 0.007 & 0 & 0.000 & 0.000 & 0.000 & 0.000 & 0.000 & 0.26 & 0 & 167 \\
\hline NGC 3079 & 3 & 0.109 & 6.278 & 0.251 & 0.028 & 0.023 & 3 & 0.213 & 6.280 & 0.368 & 0.112 & 0.207 & 0.29 & 3 & 100 \\
\hline NGC 3190 & 5 & 0.263 & 12.673 & 0.725 & 0.159 & 0.197 & 12 & 1.025 & 12.844 & 1.642 & 0.887 & 1.891 & 0.52 & 13 & 167 \\
\hline NGC 3198 & 1 & 0.033 & 1.078 & 0.036 & 0.001 & 0 & 1 & 0.033 & 0.125 & 0.004 & 0.000 & 0.000 & 0.15 & 1 & 34 \\
\hline NGC 3489 & 0 & 0.000 & 0.000 & 0.000 & 0.000 & 0 & 11 & 1.076 & 2.174 & 0.219 & 0.006 & 0.001 & 0.39 & 12 & 0 \\
\hline NGC 3516 & 2 & 0.075 & 3.821 & 0.144 & 0.012 & 0.007 & 0 & 0.000 & 0.000 & 0.000 & 0.000 & 0.000 & 0.19 & 0 & 67 \\
\hline NGC 3607 & 6 & 0.216 & 22.133 & 0.806 & 0.171 & 0.323 & 14 & 0.976 & 23.177 & 1.574 & 0.720 & 2.310 & 0.34 & 15 & 200 \\
\hline NGC 3623 & 1 & 0.142 & 1.504 & 0.213 & 0.045 & 0.031 & 7 & 1.236 & 4.603 & 1.055 & 0.399 & 0.310 & 0.44 & 7 & 34 \\
\hline NGC 3898 & 5 & 0.169 & 9.638 & 0.302 & 0.022 & 0.015 & 13 & 1.145 & 4.210 & 0.374 & 0.038 & 0.026 & 0.56 & 14 & 167 \\
\hline NGC 3921 & 20 & 0.778 & 32.854 & 1.393 & 0.175 & 0.173 & 2 & 0.476 & 1.976 & 0.311 & 0.050 & 0.027 & & 2 & 667 \\
\hline NGC 3945 & 1 & 0.024 & 1.546 & 0.037 & 0.001 & 0 & 4 & 0.450 & 1.059 & 0.090 & 0.002 & 0.000 & 0.50 & 4 & 34 \\
\hline NGC 3962 & 2 & 0.049 & 2.826 & 0.069 & 0.002 & 0.001 & 2 & 0.211 & 0.423 & 0.040 & 0.001 & 0.000 & 0.32 & 2 & 67 \\
\hline NGC 4026 & 1 & 0.051 & 1.121 & 0.057 & 0.003 & 0.001 & 22 & 2.451 & 14.406 & 1.146 & 0.354 & 0.833 & 1.71 & 23 & 34 \\
\hline NGC 4027 & & & & & & & 14 & 2.139 & 6.055 & 0.624 & 0.044 & 0.021 & 0.61 & 15 & \\
\hline NGC 4036 & 2 & 0.051 & 3.585 & 0.096 & 0.006 & 0.004 & 5 & 0.699 & 1.262 & 0.163 & 0.007 & 0.001 & 0.44 & 5 & 67 \\
\hline NGC 4111 & 8 & 0.344 & 19.254 & 0.754 & 0.093 & 0.08 & 13 & 1.138 & 25.703 & 1.337 & 0.340 & 0.754 & 1.09 & 14 & 267 \\
\hline NGC 4350 & 1 & 0.086 & 3.484 & 0.300 & 0.090 & 0.117 & 25 & 2.773 & 8.731 & 1.093 & 0.260 & 0.328 & 2.72 & 26 & 34 \\
\hline NGC 4379 & 2 & 0.111 & 3.630 & 0.215 & 0.034 & 0.026 & 34 & 2.964 & 10.198 & 0.927 & 0.055 & 0.014 & 2.89 & 36 & 67 \\
\hline NGC 4450 & 1 & 0.024 & 3.509 & 0.086 & 0.007 & 0.006 & 9 & 1.083 & 2.464 & 0.285 & 0.012 & 0.002 & 1.88 & 9 & 34 \\
\hline NGC 4565 & 5 & 0.188 & 6.516 & 0.251 & 0.017 & 0.008 & 2 & 0.363 & 2.851 & 0.400 & 0.102 & 0.101 & 1.00 & 2 & 167 \\
\hline NGC 4579 & 1 & 0.029 & 1.595 & 0.046 & 0.002 & 0.001 & 35 & 3.215 & 8.425 & 0.849 & 0.047 & 0.012 & 3.26 & 37 & 34 \\
\hline NGC 4594 & 1 & 0.022 & 1.543 & 0.034 & 0.001 & 0 & 2 & 0.203 & 0.467 & 0.044 & 0.001 & 0.000 & 0.32 & 2 & 34 \\
\hline NGC 4736 & 1 & 0.020 & 1.020 & 0.021 & 0.000 & 0 & & & & & & & 0.42 & & 34 \\
\hline NGC 4762 & 2 & 0.139 & 4.719 & 0.324 & 0.074 & 0.071 & 16 & 1.534 & 6.618 & 0.726 & 0.131 & 0.114 & 2.65 & 17 & 67 \\
\hline NGC 5018 & 5 & 0.354 & 7.384 & 0.491 & 0.116 & 0.085 & 3 & 0.871 & 2.590 & 0.696 & 0.256 & 0.250 & 0.29 & 3 & 167 \\
\hline NGC 5055 & 1 & 0.045 & 1.562 & 0.070 & 0.005 & 0.002 & 4 & 0.287 & 1.403 & 0.067 & 0.002 & 0.000 & 0.40 & 4 & 34 \\
\hline NGC 5084 & 4 & 0.306 & 7.022 & 0.463 & 0.062 & 0.038 & 7 & 0.950 & 3.186 & 0.399 & 0.035 & 0.013 & 0.29 & 7 & 134 \\
\hline NGC 5746 & 4 & 0.098 & 5.349 & 0.132 & 0.005 & 0.002 & 7 & 0.594 & 2.978 & 0.296 & 0.032 & 0.014 & 0.83 & 7 & 134 \\
\hline NGC 5846 & 3 & 0.457 & 4.665 & 0.662 & 0.261 & 0.231 & 14 & 1.862 & 28.351 & 1.264 & 0.443 & 2.149 & 0.84 & 15 & 100 \\
\hline NGC 5866 & 5 & 0.196 & 8.878 & 0.337 & 0.024 & 0.012 & 3 & 0.513 & 1.858 & 0.350 & 0.077 & 0.039 & 0.24 & 3 & 167 \\
\hline NGC 6868 & 7 & 0.491 & 14.982 & 1.007 & 0.270 & 0.43 & 10 & 1.882 & 2.565 & 0.519 & 0.041 & 0.008 & 0.47 & 11 & 234 \\
\hline NGC 7052 & & & & & & & 1 & 0.161 & 0.093 & 0.015 & 0.000 & 0.000 & & 1 & \\
\hline
\end{tabular}

to the gravitational potential and $\rho_{22}$ to the gravitational force exerted by the surrounding galaxies on the central object, whereas $\rho_{3,2.4}$ is proportional to the tidal interaction between the surrounding galaxies and the central one. The last two parameters amplify the effects present in the parameter $\rho_{11}$. These parameters were chosen in accordance with similar studies (Heckman et al. 1985; FuentesWilliams \& Stocke 1988).

After conversion into linear units, as described in the precedent paragraphs, the diameters and the distances 
from the central galaxies were converted into units of $100 \mathrm{kpc}$. The resulting values of the $\rho_{i j}$ for the field of each central galaxy are listed in Tables 2 and 3. Results from the APM database and NED are presented together in these tables.

After defining the $\rho_{i j}$ parameters for the two samples of counter-rotating galaxies and normal galaxies, a Kolgomorov-Smirnov test was applied to the $\rho_{i j}$ parameters of the local environment (APM data, galaxies within $100 \mathrm{kpc}$ ), to that of the intermediate environment (NED, bright galaxies with similar red-shift) and to $\rho_{x y z}$ densities (Tully 1988, on $40 \mathrm{Mpc}$ scale). The results are shown in Table 4.

\section{Results}

We begin by considering the local densities at three different scales: a close environment within $100 \mathrm{kpc}$, an intermediate environment inside $0.61 \mathrm{Mpc}$, defined by a crossing time of $1 \mathrm{Gyr}$, and a large scale environment inside $40 \mathrm{Mpc}$. These densities were extracted from the APM, NED and Tully (1988) catalogues respectively. The APM data refers to a projected galaxy density $\rho_{\mathrm{APM}}$, whereas the NED and Tully (1988) data use the red-shift to define "volume" densities $\rho_{\mathrm{NED}}$ and $\rho_{x y z}$ respectively. When these densities are plotted against each other (Fig. 1) we can see that $\rho_{x y z}$, and $\rho_{\mathrm{NED}}$ are correlated. In particular, excluding NGC 4379, all the remaining galaxies with gas counterrotation belong to groups where the density of galaxies $\rho_{x y z}<0.5$ galaxies $\mathrm{Mpc}^{-3}$ and $\rho_{\mathrm{NED}}<1.8$ galaxies $\mathrm{Mpc}^{-3}$. On the contrary, the projected densities extracted from APM data span the entire range of plotted values, without any particular clustering of points. This may be affected by the presence of background objects, which alter the sample, despite our selection criteria defined in Sect. 4, or may suggest that there is no particular clustering of objects in the surrounding of our sample galaxies. However, bearing in mind that our cut-off levels in magnitudes and sizes for possible companion galaxies were quite high, it is more likely that a few satellites were missed rather than having a significant contamination by background objects. We think then that the plot reflects the real situation of the densities existing within $100 \mathrm{kpc}$ of our sample objects.

This segregation/concentration for galaxies with gas counterrotation in Fig. 1 is confirmed by a KolgomorovSmirnov test applied to the galaxy densities around the whole sample, except for NGC 4379. The population of galaxies with gas counterrotation and the population of normal galaxies appear to be different at a significance level of $93.7 \%$. However, there is no a priori justification for the exclusion of NGC 4379. Its inclusion reduces the significance level to $84 \%$ and weakens the difference between populations. The same test applied to the NED and APM densities indicates that the difference between samples decreases when smaller environments are considered (see the last lines of Table 4). We may conclude that, in the limited number of galaxies with pure gas

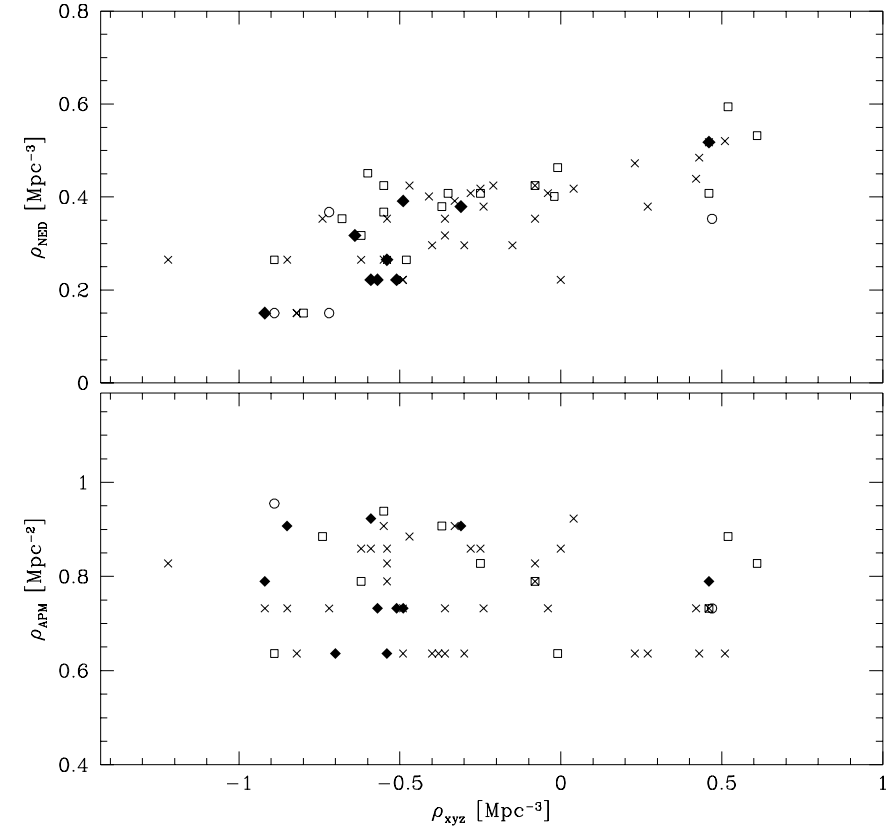

Fig. 1. Plot of the density of objects around all the sample galaxies in environments with different size. The galaxies with pure gas counterrotation or pure stellar counterrotation are indicated by full diamonds and open squares respectively; the normal galaxies are indicated by crosses. The galaxies with both gas and stellar counterrotation are plotted with open circles. Top panel: plot of $\left(\rho_{\mathrm{NED}}\right)$ the density of galaxies $/ \mathrm{Mpc}^{3}$ present in NED database with a crossing time lower than $1 \mathrm{Gyr}$ versus $\left(\rho_{x y z}\right)$, the density of galaxies computed within $40 \mathrm{Mpc}$ from Tully (1988). Bottom panel: plot of $\left(\rho_{\mathrm{APM}}\right)$, the density of galaxies extrapolated to a square of $1 \mathrm{Mpc}$ side on the sky, versus $\left(\rho_{x y z}\right)$. These plots are discussed in the text.

counterrotation available, they tend to lie in less dense groups, on scales larger than $\sim 0.5 \mathrm{Mpc}$.

Looking at the other studied parameters, described by the $\rho_{i j}$ quantities (Tables 2 and 3), Kolgomorov-Smirnov tests indicate that no marked differences are evident in APM or in the NED data. In fact, no significance level is above $\sim 80 \%$ (Table 4 ).

\section{Conclusions}

We deduce that in general the surrounding regions of galaxies with counterrotation do not appear statistically different from those of normal galaxies. This result is similar to that found in Paper I for the environment of polar ring galaxies but distinguish our galaxies from the other active galaxy categories (Dahari 1984; Heckman et al. 1985; Hintzen et al. 1991; Rafanelli et al. 1995).

Among the hypotheses presented in the Introduction about the origin of counterrotation and polar rings, our result tend to disprove that of a recent interaction with a small satellite or a galaxy with similar size. If such a process is at the origin of the counterrotation phenomenon (Balcells \& Gonzalez 1998; Bekki 1998; Kennicut 1996; Thakar \& Ryden 1996), it cannot be younger than 1 Gyr, the crossing time for the volumes of space studied in this 
Table 4. Summary of Kolgomorov-Smirnov tests on the density parameters calculated from the data extracted from APM and NED databases. $D_{\alpha}$ is the maximum difference observed between the two distributions, whereas $S L$ is the percentage significance level at which the two distributions compared are different. In the last three lines, we show the results of the tests applied to the density $\rho_{x y x}$ and $\rho_{\mathrm{NED}}$ (in galaxies $/ \mathrm{Mpc}^{3}$ ) and $\rho_{\text {APM }}$ (in galaxies $/ \mathrm{Mpc}^{2}$ ) described in the text.

\begin{tabular}{|c|c|c|c|c|c|c|}
\hline \multirow[b]{2}{*}{ Param. } & \multicolumn{2}{|c|}{ gas cr vs. no $\mathrm{cr}$} & \multicolumn{2}{|c|}{ stars cr vs. no $\mathrm{cr}$} & \multicolumn{2}{|c|}{ all $\mathrm{cr}$ vs. no $\mathrm{cr}$} \\
\hline & $D_{\alpha}$ & $S L$ & $D_{\alpha}$ & $S L$ & $D_{\alpha}$ & $S L$ \\
\hline \multicolumn{7}{|c|}{ APM (100 kpc environment) } \\
\hline$\rho_{00}$ & 0.178 & $13.3 \%$ & 0.292 & $78.6 \%$ & 0.217 & $64.9 \%$ \\
\hline$\rho_{01}$ & 0.283 & $66.9 \%$ & 0 & & & $72.9 \%$ \\
\hline & 0.257 & $54.9 \%$ & 0.23 & $3 \%$ & 97 & $58.8 \%$ \\
\hline$\rho_{11}$ & 0.207 & $28.5 \%$ & 0.179 & $20.9 \%$ & 0.179 & $40.6 \%$ \\
\hline$\rho_{22}$ & 0.145 & $<10 \%$ & 0.121 & $<10 \%$ & 0.096 & $<10 \%$ \\
\hline$\rho_{3,2.4}$ & 0.171 & $10.4 \%$ & 0.079 & $<10 \%$ & 0.050 & $<10 \%$ \\
\hline \multicolumn{7}{|c|}{ NED (1 Gyr similar redshift companions) } \\
\hline$\rho_{00}$ & 0.167 & $12.7 \%$ & 0.130 & $<10 \%$ & 0.056 & $<10 \%$ \\
\hline$\rho_{01}$ & 0.206 & $34.6 \%$ & 0.242 & $67.9 \%$ & 0.166 & $40.1 \%$ \\
\hline$\rho_{10}$ & 0.071 & $<10 \%$ & 0.129 & $<10 \%$ & 0.058 & $<10 \%$ \\
\hline & 0.135 & $<10 \%$ & 0.129 & $<10 \%$ & 0.095 & $<10 \%$ \\
\hline & 0.111 & $<10 \%$ & 0.150 & $12.8 \%$ & 0.097 & $<10 \%$ \\
\hline$\rho_{3,2.4}$ & 0.056 & $<10 \%$ & 0.154 & $15.4 \%$ & 0.097 & $<10 \%$ \\
\hline \multicolumn{7}{|c|}{ densities of galaxies from different catalogues } \\
\hline & 0.372 & $84.2 \%$ & 0.140 & $<10 \%$ & 0.238 & $71.6 \%$ \\
\hline & 0.206 & $34.6 \%$ & 0.155 & $15.9 \%$ & 0.063 & $20.4 \%$ \\
\hline$\rho_{\mathrm{APM}}$ & 0.178 & $13.3 \%$ & 0.203 & $34.8 \%$ & 0.153 & $22.3 \%$ \\
\hline
\end{tabular}

paper. Otherwise it is difficult to conceive that no trace of the donor galaxy remains in the surrounding space, both as a single galaxy present in the NED archive or in a form detectable in APM data as diffuse surrounding objects.

This result, to a first approximation, support the hypothesis that all galaxies are born from a merger process of smaller objects occurring early in their life. However, only a few galaxies that we know of develop counterrotation and polar rings. It may be natural to attribute this peculiarity to a richer environment, which makes the possibility of collisions easier. Our data are also contrary to this hypothesis, because the environment of such galaxies does not appear to be richer in satellites. This is different to Seyfert or radio-loud galaxies which lie in environments with a higher density of companions. On the contrary, if a weak tendency exists for galaxies with gas counterrotation only, it is seen in regions of space where the large scale density of galaxies is smaller. Whatever the special machinery is which produces counterrotation or polar rings instead of a co-planar, co-rotating distribution of gas and stars, it is not connected to the present galaxy density of their environments.

An alternative mechanism to form counterrotation and polar rings may arise from a continuous, non traumatic infall of gas that later formed stars (Voglis et al. 1991; Quinn \& Binney 1992; Merrifield \& Kuijken 1994; Ostriker \& Binney 1989; Rix et al. 1992). In such a case the past and present visible environment of these galaxies would appear similar to that of the other galaxies, even if the process is still active. This explanation is consistent with all the results we found. The slow infall of matter on a galaxy should not alter either its luminosity distribution or its stellar kinematics, untill the accreted mass is large enough to generate tidal actions. The galaxies with counterrotation and the polar rings may in such a scenario appear relaxed or in equilibrium, even if some star formation is active (see the polar rings of NGC 4650A and NGC 5128).

The study of the peculiar galaxies that present gas accretion is still under discussion of the possible models to explain their origin and evolution. It is currently impossible to decide between the previous, perhaps incompatible, theories (early merging with special dynamical conditions or continuous slow infall). On the other hand, the observations of galaxies with counterrotation, begun in 1984, provide clues to their evolution, but are still not conclusive. To solve the problem, we are planning to study the gas content of galaxies with counterrotation and polar rings (Bettoni et al. 2001).

Acknowledgements. We would like to thank Dr. J. Sulentic for useful suggestions regarding this paper. This research was done using the LEDA database, (leda.univ-lyon1.fr), of the NASA/IPAC Extragalactic Database (NED, nedwww.ipac.caltech.edu) which is operated by the Jet Propulsion Laboratory, California Institute of Technology, under contract to the National Aeronautics and Space Administration and of the Hypercat database (www-obs.univ-lyon1.fr/hypercat). This search has been granted by the funds $60 \%-2000$ of the Università di Padova.

\section{References}

Balcells, M., \& Gonzalez, A. C. 1998, ApJ, 505, L109

Barnes, J. E. 1992, ApJ, 393, 484

Bekki, K. 1998, ApJ, 499, 635

Bertola, F., Cinzano, P., Corsini, E. M., et al. 1996, ApJ, 458, L27

Bettoni, D. 1984, The Messenger, 37, 17

Bettoni, D. 1989, AJ, 97, 79

Bettoni, D., Fasano, G., \& Galletta, G. 1990, AJ, 99, 1789

Bettoni, D., Galletta, G., \& Oosterloo, T. 1991, MNRAS, 248, 544

Bettoni, D., Galletta, G., Garcia-Burillo, S., \& RodriguezFranco, A. 2001, A\&A, in press

Bettoni, D., Galletta, G., \& Sage, L. J. 1999, AA, 280, 121

Bettoni, D., Galletta, G., Garcia-Burillo, S., \& RodriguezFranco, A. 1999, in Galaxy Dynamics: from the Early Universe to the Present, ed. F. Combes, G. A. Mamon, \& V. Charmandaris, ASP Conf. Ser., 197, 89

Bender, R. 1988, AA, 202, L5

Brocca, C., Bettoni, D., \& Galletta, G. 1997, A\&A, 326, 907, Paper I

Caldwell, N., Kirshner, R. P., \& Richstone, D. O. 1986, ApJ, 305,136

Ciri, R., Bettoni, D., \& Galletta, G. 1995, Nature, 375, 661

Corsini, E. M., \& Bertola, F. 1998, J. Corean Phys. Soc., 33, 574 
Dahari, O. 1984, AJ, 89, 966

Fuentes-Williams, T., \& Stocke, J. T. 1988, AJ, 96, 1235

Franx, M., \& Illingworth, G. D. 1988, ApJ, 327, L55

Galletta, G. 1987, ApJ, 318, 531

Galletta, G. 1996, in Barred Galaxies, IAU Coll. 117, ed. R. Buta, D. A. Crocker, \& B. G. Elmegreen, ASP Conf. Ser., 91,429

Garcia, A. M., Paturel, G., Bottinelli, L., \& Gouguenheim, L. 1993, A\&AS, 98, 7

Gunn, J. E., 1979, Active galactic nuclei (A79-50785 22-90) Cambridge (Cambridge University Press), 213

Heckman, T. M., Carty, T. J., \& Bothun, G. D. 1985, ApJ, 288, 122

Hintzen, P., Romanishin, W., \& Valdes, F. 1991, ApJ, 366, 7

Irwing, M., Maddox, S., \& Mc Mahon, R. 1994, Spectrum, 2, 14

Jedrzejeswski, R., \& Schecther, P. L. 1988, ApJ, 330, L87

Kennicutt, R. 1996 in New light on galaxy evolution, IAU Symp. 171, ed. R. Bender, \& R. L. Davies, 11

Kuijken, K., Fisher, D., \& Merrifield, M. R. 1996, MNRAS, 283,543

Merrifield, M. R., \& Kuijken, K. 1994, ApJ, 432, 575

Ostriker, E. C., \& Binney, J. J. 1989, MNRAS, 237, 785

Paturel, G., Bottinelli, L., Di Nella, H., et al. 1997, A\&AS, 124,109

Prada, F., Gutierrez, C. M., Peletier, R. F., \& McKeith, C. D. 1996, ApJ, 463, L9
Prada, F., \& Gutierrez, C. M. 1999, ApJ, 517, 123

Prugniel, Ph., Zasov, A., Busarello, G., \& Simien, F. 1998, A\&AS, 127, 117

Quinn, P. J., Hernquist, L., \& Fullagar, D. P. 1993, ApJ, 403, 74

Quinn, T., \& Binney, J. 1992, MNRAS, 255, 729

Rafanelli, P., Violato, M., \& Baruffolo, A. 1995, AJ, 109, 1546

Reshetnikov, V., \& Sotnikova, N. 1997, A\&A, 325, 933

Rix, H.-W., Franx, M., Fisher, D., \& Illingworth, G. 1992, ApJ, 400, L5

Rubin, V. C., Graham, J. A., \& Kenney, J. D. P. 1992, ApJ, 394, L9

Sandage, A. R., \& Tamman, G. A. 1981, A revised ShapleyAmes Catalog of Bright Galaxies, Carnegie Inst. of Washington, Washington, DC

Thakar, A. R., \& Ryden, B. S. 1996, ApJ, 461, 55

Tremaine, S., \& Yu, Q. 2000, MNRAS, 319, 1

Tully, R. B. 1987, ApJ, 321, 280

Tully, R. B. 1988, Nearby Galaxies Catalog (Cambridge University Press, Cambridge, UK)

Voglis, N., Hiotelis, N., \& Höfflich, P. 1991, A\&A, 249, 5

Whitmore, B. C., Lucas, R. A., McElroy, D. B., et al. 1990, AJ, 100, 1489

Zombeck, M. V. 1990, Handbook of Astronomy and Astrophysics, Second Edition (Cambridge, UK: Cambridge University Press) 